\title{
Arrhythmias caused by chemotherapy agents used on the treatment of triple- negative Breast cancer: A mini review of literature
}

\author{
Maíra Lyra Teles ${ }^{1}$, Luiza Silva La Rocca de Freitas ${ }^{1}$, Luísa Teixeira Fischer Dias ${ }^{1}$, Letícia Ferreira \\ Gomes Vasconcelos ${ }^{1}$ and Antoinette Oliveira Blackman ${ }^{2 *}$ \\ ${ }^{1}$ Medical Student in Centro Universitário do Planalto Central Aparecido dos Santos UNICEPLAC, Brazil \\ ${ }^{2}$ Professor at medical school UNICEPLAC, Department of Medicine, Brazil
}

*Corresponding author: Antoinette Oliveira Blackman, Professor of Medical School, Department of Medicine, University of Research, UNICEPLAC, Brazil

\section{ARTICLE INFO}

Received: May 05, 2020

Published: May 14, 2020

Citation: Maíra Lyra T, Luiza Silva La Rocca de F, Luísa Teixeira FD, Letícia Ferreira GV, Blackman AO. Arrhythmias caused by chemotherapy agents used on the treatment of triple- negative Breast cancer: A mini review of literature. Biomed J Sci \& Tech Res 27(4)-2020. BJSTR. MS.ID.004536.

Abbreviations: 5-FU: 5-Fluorouracil; AV: Atrioventricular; AF: Atrial Fibrillation; BC: Breast Cancer; CAD: Coronary Artery Disease; CTX: Cyclophosphamide; DTX: Docetaxel; ECG: Electrocardiogram; EPI: Erupicin; FDA: Food and Drug Administration; LVEF: Left Ventricular Ejection Fraction; PTX : Paclitaxel ; PVCs: Premature Ventricular Complexes; SCD: Sudden Cardiac Death; SVT: Supraventricular Tachycardia; VF: Ventricular Fibrillation; VT: Ventricular Tachycardia; TdP: Torsades de Pointes; TNBC: Triple-Negative Breast Cancer

\section{Abstract}

Introduction/Background: Triple negative breast cancer (TNBC) represents a significant portion of the types of breast cancer. With the worst prognosis, TNBC is treated with polychemotherapy, increasing patient survival and, consequently, causing cardiotoxic effects and electrocardiographic changes.

Method: This study is a literature review realized in Public Medline (PubMed) as a total, 11 articles were used dated between January 2015 and April 2020.

Results: The ECG changes of anthracyclines are prolongation of the QT interval and ventricular arrhythmias, increased with higher doses. In alkylating agent's ECG changes identified are atrial complexes, atrial fibrillation, QT interval prolongation and AV block. Detected changes in the antimetabolites group are ST-T wave changes, prolonged $\mathrm{P}$ wave duration, increased $\mathrm{P}$ wave dispersion, QT interval prolongation, QT dispersion and diffusely decreased QRS voltage. Antimicrotubule agents, changes found are T-wave changes, left Bundle block, right Bundle block, AV block (rare cases) and QT interval prolongation.

Discussion: Therefore, the broad spectrum of arrhythmias caused by chemotherapy treatment, it is essential to monitor these patients before, during and after the use of these drugs. and to adopt preventative measures, such as dexrazoxane in order to avoid arrhythmias caused by doxorubicin, as well antihistamine and corticosteroid to prevent them caused by paclitaxel. CONCLUSION: Survival cancer patients should be better monitored with ECG and 24-hr Holter so that arrhythmias can be untimely detected, thus decreasing morbidity and mortality.

Keywords: Breast cancer; Chemotherapy; Cardiotoxicity; Electrocardiography; Arrhythmias

\section{Introduction}

Breast cancer (BC) is the most common type of cancer in women after skin cancer. Triple-negative breast cancer (TNBC) corresponds to $15 \%$ of $\mathrm{BC}$. TNBC mortality in five years floats between $30 \%$ to $40 \%$ [1-2]. The group formed by TNBC is not homogenic, although exhibits some biologic mechanisms, as high proliferation and poor differentiation that brings worse prognosis than hormone responsive $\mathrm{BC}$ [2]. As a result, this kind of breast cancer was treated with polychemotherapy, and sometimes receiving special drugs. The most common chemotherapy drugs used in TNBC are Erupicin (EPI), cyclophosphamide (CTX), paclitaxel (PTX), docetaxel (DTX), doxorubicin, 5-fluorouracil (5-FU). Over the years, the number of cancer survivors and the use of different chemotherapy agents 
has increased, as well as the cumulative dose of chemotherapies, and this implicates in risk for adverse cardiovascular events [2]. Those can be divided into two groups: acute, as coronary artery syndrome, pericarditis, myocarditis, cardiac arrhythmias, or QT interval prolongation; or chronic cardiotoxicity as result of reduced left ventricular ejection fraction (LVEF), heart failure (HF) or death [1-2].

Cardiotoxicity chemotherapy-induced is defined by declining LVEF and symptoms of HF and it is commonly discussed in terms of cardiac dysfunction. Only recently cancer-treatment inducing arrhythmia (CTIA), and changes in ventricular repolarization have been brought to attention [1,3-5]. With the aging of the population, both cardiac diseases and cancer have turned into more prevalent diseases, therefore, cardiotoxicity is likely to occur in many patients undergoing cancer treatment. Contrary to initial guesses, the risk for cardiotoxicity still happens with the new target therapy agents, which act by inhibiting specific signals, such as monoclonal antibodies (trastuzumabe and pertuzmabe) [3-6] Therefore, CTIA can be divided into primary and secondary. Primary CTIA occurs when a drug has an effect interfering in a molecular pathway that causes an specific arrhythmia. Secondary CTIA is due to a kind of damage to the heart tissues leading the arrhythmia, being the most common one [3]. The real incidence of CTIA is not known due to many factors. Most cancer treatment trials exclude patients with cardiovascular diseases, which are some of the patients that will more likely develop cardiotoxic events [3-4,6-7].

Also, baseline ECGs are not taken before the beginning of trials, which makes it harder to know if the arrhythmia is indeed caused by the chemotherapy treatment. In addition, most patients are treated with multiple chemotherapy drugs protocols which also makes it difficult to establish the drug that causes the cardiotoxicity effect $[3-4,7]$. Furthermore, it is difficult to determine the exact chemotherapy agent that developed the arrhythmia because cancer and cardiac diseases have many risk factors in common, for example, obesity and alcohol use [3] Risk factors associated with cardiotoxicity of chemotherapeutic agents are broad, which included history of coronary artery disease (CAD), diabetes mellitus (DM), hypertension (HTN), arrhythmias, history of congestive heart failure ( $\mathrm{CHF}$ ), congenital heart disease, chest radiation, exposure to cardiotoxic chemotherapy, drug abuse, pulmonary hypertension (PHTN), pulmonary changes such as asthma, sleep apnea, sarcoidosis, thyroid dysfunction, chronic kidney disease and miscellaneous causes that included any other rare disease processes that could potentially cause CHF. Common factors found in breast cancer patients, according to age group [5,8]. In this context, anthracyclines (doxorubicin and epirubicin) present risk of adverse events is significantly elevated in the cumulative dose. Other agents can cause cardiac damage via different pathophysiological mechanisms, such as: monoclonal antibodies (trastuzumab and pertuzumab), antimetabolite drugs (5-Fluorouracil), alkylating agents (cyclophosphamide) and antimicrotubule agents (paclitaxel and docetaxel). It is important to monitor patients on chemotherapy treatment because myocardial injury induced by the drug reduces the survival in patients who outlasted cancer by $50 \%$ in 5 years [9].

Multiple factors, including drugs and other conditions, for example: electrolyte abnormalities (hypokalemia or hypomagnesemia), bradycardia, LV hypertrophy and CHF,HIV infection, intracranial pathologic abnormality, connective tissue disorders, hypothermia, age, genetic disorders and gender can modify QT interval and cause arrhythmia [1,6]. This finding can flare arrhythmia and has been associated with polymorphic ventricular tachycardia, torsades de pointes (TdP) [1,6]. On a surface electrocardiogram (ECG) the QT interval is measured from the beginning of the QRS complex to the end of the T wave. Normal depolarization occurs due to a rapid inflow of sodium and calcium, whereas repolarization is due to outflow of potassium ions. Most drugs that prolong QT interval impact the function of the K channel typically by inhibiting or modulating the function. The measurement of the QT interval it's difficult because it can be particularly challenging to determine the end of the $\mathrm{T}$ wave $[1,6]$. Although QT interval prolongation is a common effect of chemotherapy agents, TdP is rarely seen. Nevertheless, due to its life-threatening potential, it's important to measure the QT interval, as it is the best available marker to quantify the risk of developing this arrhythmia [3]. However, there are few studies regarding CTIA, essentially about QT interval prolongation. Therefore, the aim of this study is to indicate the necessity of careful monitoring with ECG records in TNBC patients with neoadjuvant or adjuvant chemotherapy to screen high-risk groups for chemotherapy-induced cardiac events and improve treatment decision for these patients.

\section{Materials and Methods}

This study is a literature review realized in Public Medine (PubMed) using the key words "breast cancer", "chemotherapy", "cardiotoxicity" "electrocardiography" between January 2015 and April 2020. The articles

I. Selection Criteria:

a. Describe pathophysiology of prolonged QTc induced by chemotherapy;

b. Explain mechanism of cardiotoxicity;

c. Demonstrate side effects of breast cancer treatment.

II. Exclusion Criteria:

a. Have as its main subject other chemotherapy agents;

b. Did not refer to cardiotoxicity in electrocardiography alterations;c.

c. Elucidated only about animal models;

d. Did not refer to QT interval prolongation. As a total, 11 articles were used. 


\section{Discussion}

Based on the articles found and in the context of BC treatment, the classes of chemotherapeutic agents most used that are related to CTIA are anthracyclines, alkylating agents, antimicrotubule agents, antimetabolites and HER2 inhibitors (monoclonal antibodies). Cardiotoxicity due to the use of anthracyclines occurs due to the perpetuation of cell damage caused by necrosis and apoptosis that is not repaired in a timely manner. The number of cardiomyocytes decreases causing remodeling and leading overt symptoms. Resulting in transient electrocardiographic changes, LV dysfunction, myocarditis and supraventricular arrhythmias [5, 9-10]. There are many mechanisms proposed to explain the cardiotoxic effects of the anthracyclines $[1,4,6]$. Damage to cardiac tissues can be demonstrated by the production of free radicals mediated by iron-dependent enzymes, leading to the production of superoxide in the myocardium, in addition to apoptosis and necrosis of cardiac tissue after exposure to chemotherapy. Frequent cardiac remodeling with diastolic dysfunction is an early indicator of cardiotoxicity and development of posterior systolic dysfunction, being identified as an injury in cardiomyocytes [1,4, 6, 9-10].

Other mechanisms include direct electrophysiological effects caused by the increase of the amplitude of L-typeCa2+ currents observed in rats treated with doxorubicin for a long time; in canine the amplitude is decreased and the cardiac action potential is prolonged of the Purkinje system, among others [4]. Doxorubicin and epirubicin are anthracyclines used to treat breast cancer and the most frequent cardiotoxic effect of this class of chemotherapy agents is cardiomyopathy; CTIA can occur as a secondary effect to this dysfunction, but also as a primary CTIA $[1,3,6]$. The cardiotoxicity is dose dependent and not reversible [3]. Due to its cardiotoxic potential, anthracyclines should be carefully administered [10]. The alterations seen in ECGs are generally nonspecific, occur even during the first infusion of these drugs and are found in less than $30,3 \%$ of patients. The findings can include T-wave flattening, alterations in ST-T segment, lowering of the QRS voltage and prolongation of QT interval. The prolongation of QT interval is common with QT $>450 \mathrm{~ms}$ identified in the first cycle of the treatment in $11,5 \%$ of patients. Although low LVEF is the most recognized cardiotoxic effect of the anthracyclines, QT interval prolongation is seen independent of $\mathrm{LV}$ function. With higher doses and sequential cycles, it can lead to ventricular arrhythmias [3-4,6] Other arrhythmias observed are sinus bradycardia or tachycardia, supraventricular tachycardia (SVT), ventricular tachycardia (VT), premature ventricular complexes (PVCs), premature atrial complexes and bradyarrhythmias, including atrioventricular (AV) block.

A study showed that the incidence of these findings are low in the first hour of infusion (3\%) but rise after that period, reaching $24 \%$ in the first 24 hours, although most of them are benign. It's relevant to monitor and maintain electrolyte levels at its normal, since hypokalemia can worsen these arrhythmias [3-4,6]. There are reports of QT interval prolongation and TdP causing sudden cardiac death (SCD) in patients with hypokalemia and VT or ventricular fibrillation (VF) [4]. The risk factor for developing these arrhythmias are extremes of age (less than 15 or more than 65 years old), pre-existing cardiovascular diseases, female sex, cumulative doses, hypokalemia, concomitant use of other chemotherapy agents, mediastinal irradiation and liver or renal dysfunction $[4,6]$. There are ways to early detect and prevent these arrhythmias, as ECG monitoring before, during and after the use of doxorubicin, treatment of cardiovascular risk factors, avoiding administering doxorubicin along with other chemotherapy agents [4]. It is described that dexrazoxane, an iron-chelating agent approved by the Food and Drug administration (FDA), can reduce QT interval prolongation and prevent drug-induced cardiomyopathy. It acts by preventing generation of anthracycline-iron complexes and free radicals [3-4]. Patients that used anthracyclines are in long-term risk for developing these arrhythmias, even when they discontinued the drug, thus it is recommended that a follow up is proposed with ECG monitoring so life-threatening changes can be detected $[4,6]$.

Cyclophosphamide is an example of an alkylating agent used to treat breast cancer. CTIA is one of the most frequent cardiotoxic events caused by this class [3]. The mechanism of action associated with these arrhythmias are coronary artery vasospasm, vascular coronary endothelial damage along with intracapillary microthrombi. The occurrence of AV block associated with vomiting in some patients suggests that increased vagal tone contributes to this arrhythmia [4,9]. Atrial tachyarrhythmias as well as ventricular tachyarrhythmias are seen in 7,9 to $10 \%$ of patients in the next 24 to 72 hours after the use of cyclophosphamide in high doses. They are specially observed in patients with pericarditis and congestive heart failure and disappear after 1 to 7 days [4] Cyclophosphamide itself can cause pericarditis, myocarditis and heart failure [9]. ECG changes identified are atrial complexes, atrial fibrillation (AF), PVCs, SVT, QT interval prolongation and AV block. A study showed that arrhythmias, alterations of ST segment, QT interval prolongation, T-wave changes and QT interval prolongation were demonstrated in $33 \%$ of the patients that used cyclophosphamide before bone marrow transplantation [3]. The risk of the need to use cardioversion and antiarrhythmic drugs increases in higher doses in patients with cardiomyopathy or chronic kidney disease [4].

The main risk factors for these arrhythmias are female sex, age over 70, hypertension, coronary artery disease (CAD), chronic kidney disease, heart failure, myocarditis and mediastinal irradiation [4]. Placlitaxel is a chemotherapy agent used in the treatment of breast cancer and the most frequently identified arrhythmia is sinus bradycardia, occurring in $29 \%$ of patients using it as a monotherapy [3-4]. In animal models, paclitaxel causes the discharge of histamine which produces bradycardia, conduction delay through the Purkinje and de AV node, furthermore the $\mathrm{H} 2$ receptor causes ventricular ectopic automaticity and induces faster 
rhythm. In addition, it increases myocardial oxygen demand at the time that reduces coronary blood flow, which produces ischemic arrhythmia, among other mechanisms [4]. The sinus bradycardia is usually moderated and asymptomatic. Another common arrhythmia is first-degree $\mathrm{AV}$ block, observed in $25 \%$ of the patients. Other arrhythmias identified are T-wave changes, left bundle block, right bundle block, AV block (rare cases) and QT interval prolongation [3-4]. Arrhythmias appear in up to 24 hours of the administration of the drug and it disappears spontaneously over the next 24 to 72 hours. Some patients have experienced SVT and PVCs in up to 10 days after paclitaxel has been discontinued [4].

The risk factors for developing these ECG changes history of bradycardia, previous AV block, congestive heart failure, bundle branch block, myocardial infarction, and the use of AV blocking drugs. Despite that, placlitaxel exacerbates the cardiotoxic effect of doxorrubicin, therefore it is recommended to be administered before paclitaxel and the infusions to have an interval of 24 hours. The use of antihistamines and corticosteroids before starting treatment decreases the histamine release, thus the risk of bradycardia. Most patients have asymptomatic arrhythmias. In symptomatic, ECG monitoring is recommended, and it should be considered the discontinuation of the chemotherapeutic. Some patients can need pacemaker implantation [4]. 5-Fluorouracil (5FU) is commonly used to treat breast cancer and the most common cardiotoxic events identified are angina and myocardial infarction, CTIA is the second most common event of this chemotherapy agent [3]. The most frequent arrhythmias observed are secondary to ischemia [3-4]. The mechanism of action of the ischemia involves coronary vasospasm which is due to the toxic effect of endothelium nitric oxide synthase and vasoconstriction caused by protein kinase C. Due to changes in the coagulation system, thrombosis can also lead to ischemia. Seric endothelin-1 levels increase in patients using 5-FU, a vasoconstrictor, which corroborates with the role of ischemia in these patients [4].

The frequently detected findings are ST-T wave changes, prolonged $\mathrm{P}$ wave duration, increased $\mathrm{P}$ wave dispersion, QT interval prolongation, QT dispersion and diffusely decreased QRS voltage [3]. Also, chest pain and creatinine kinase-MB elevations are seen in these patients. Up to $18 \%$ of the ones treated with high doses of 5-FU develop these ECG changes [4]. The incidence of VT can be up to $7,4 \%$ and it occurs as a secondary CTIA due to myocardial ischemia. 5-FU rarely causes supraventricular arrhythmias, but ventricular arrhythmias, for instance PVCs, TdP and sudden cardiac death (SCD). It can also lead to sinus bradycardia in up 11,96\% of patients, AV block and intraventricular conduction delays [34]. ECG changes caused by ischemia occur in 2 to 5 days after the administration of the antimetabolites and disappear in up to 5 days after discontinuation. However, $90 \%$ of the patients re-exposed can have reappearance of these arrhythmias, which corroborates with the cardiotoxic effect of these chemotherapy agents [4]. The risk factors for developing these arrhythmias are elderly, renal failure,
CAD, concomitant cisplatin treatment, mediastinal radiation, high doses and continuous infusion. It's also important to monitor electrolyte levels, as hypomagnesaemia increases the risk of these changes [4].

ECG findings can vary from mild to deadly. Sinus bradycardia can be caused by cyclophosphamide, 5-FU, paclitaxel and taxanes. Sinus tachycardia can be observed with cyclophosphamide, 5-FU, paclitaxel and epirubicin. AV block is detected with the use of cyclophosphamide, doxorubicin, epirubicin, 5-FU and taxanes. $\mathrm{AF}$ is caused by cyclophosphamide, doxorubicin, and taxanes. Supraventricular tachycardias can happen with cyclophosphamide, doxorubicin and taxanes. Premature ventricular contractions with doxorubicin, taxanes and 5-FU. VT or VF can be seen with cyclophosphamide, 5-FU, doxorubicin, trastuzumab and taxanes. QT interval prolongation with doxorubicin, cyclophosphamide, 5-FU and paclitaxel. Torsades de pointes with anthracyclines and 5-FU. In addition, SCD was observed with the use of 5-FU and doxorubicin [1-11]. Therefore, with the broad spectrum of arrhythmias caused by chemotherapy treatment, it is essencial to monitor these patients before, during and after the use of these drugs. ECG is an efficient, inexpensive exam that can detect these changes untimely so that measures can be implemented preventing life-threatening arrhythmias. 24-hr Holter recording is another method to evaluate more carefully the changes observed in a conventional ECG. It is pivotal to outline trials that reflect the real consequences and incidence of the arrhythmias caused by chemotherapy agents, including patients with cardiovascular risks, as they are the most susceptible group to develop these events. For this achievement, oncologists and cardiologists should work together and define strategies that can benefit patients and balance the risk of using chemotherapeutic agents $[1,4,6]$. Also, it is important to shed light on dexrazoxane in order to avoid arrhythmias caused by doxorubicin, and antihistamine and corticosteroid to prevent arrhythmias caused by paclitaxel.

\section{Conclusion}

Cancer treatment drugs can lead to ECG changes and as a result, life-threatening arrhythmias. With increased survival of cancer patients, arrhythmias caused by chemotherapy agents, mainly QT prolongation and fatal rhythms, should be closely observed. Given that they can cause long term changes and interfere with the quality of life of the patients that had survived from cancer, the focus should be in predicting these rhythms by better understanding the mechanisms of these arrhythmias and monitoring with noninvasive methods, such as ECG and 24-hr Holter. Consequently, it will be possible to decrease morbidity and mortality of these patients, therefore improving quality of life.

\section{References}

1. Veronese P, Hachul DT, Scanavacca MI, Hajjar LA, Wu TC, et al. (2018) Effects of anthracycline, cyclophosphamide and taxane chemotherapy on QTc measurements in patients with breast cancer. PLoS ONE.13(5): e0196763. 
2. Liu B, An T, Li M, Yi Z, Li C, et al. (2018) The association between early onset cardiac events caused by neoadjuvant or adjuvant chemotherapy in triple-negative breast cancer patients and some novel autophagyrelated polymorphisms in their genomic DNA: a real-world study. Cancer communications (London, England) 38(1): 71.

3. Buza V, Rajagopalan B, Curtis AB (2017) Cancer Treatment-Induced Arrhythmias: Focus on Chemotherapy and Targeted Therapies. Circ Arrhythm Electrophysiol 10(8): e005443.

4. Tamargo J, Caballero R, Delpón E (2015) Cancer chemotherapy and cardiac arrhythmias: a review. Drug Saf 38(2): 129-152.

5. Peddi P, Master SR, Dwary AD, Ravipati HP, Patel AH, et al. (2019) Utility of routine pretreatment evaluation of left ventricular ejection fraction in breast cancer patients receiving anthracyclines. Breast J 25(1): 62-68.

6. Fradley MG, Moslehi J (2017) QT Prolongation and Oncology Drug Development. Card Electrophysiol Clin 7(2): 341-355.

7. Parve S, Aliakberova GI, Gylmanov AA, Abdulganieva DI (2017) Role of Exogenous Phosphocreatine in Chemotherapy-induced Cardiomyopathy. Rev Cardiovasc Med 18(2): 82-87. PMID: 29038417

ISSN: 2574-1241

DOI: $10.26717 /$ BJSTR.2020.27.004536

Oliveira Blackman A. Biomed J Sci \& Tech Res

(C) This work is licensed under Creative

Submission Link: https://biomedres.us/submit-manuscript.php
8. Papakonstantinou A, Matikas A, Bengtsson NO, Malmström P, Hedayati E, et al. (2020) Efficacy and safety of tailored and dose-dense adjuvant chemotherapy and trastuzumab for resected HER2-positive breast cancer: Results from the phase 3 PANTHER trial. Cancer 126(6): 11751182.

9. De Iuliis F, Salerno G, Taglieri L, De Biase L, Lanza R, et al. (2016) Serum biomarkers evaluation to predict chemotherapy-induced cardiotoxicity in breast cancer patients. Tumour Biol 37(3): 3379-3387.

10. Inanc MT, Karadavut S, Aytekin M, Duran AO, Derya M, et al. (2016) The relationship between plasma hyaluronan levels and anthracyclinerelated cardiotoxicity in breast cancer patients. Int J Cardiol 218: 246251.

11. Demissei BG, Finkelman BS, Hubbard RA, Smith AM, Narayan HK, et al (2019) Cardiovascular Function Phenotypes in Response to Cardiotoxic Breast Cancer Therapy. J Am Coll Cardiol 73(2): 248-249.

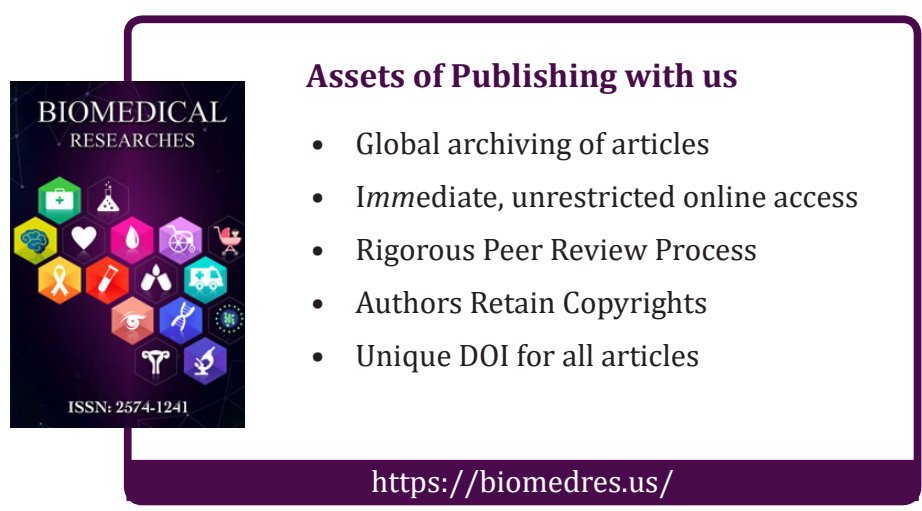

